\title{
Volcanic gas release rates
}

\section{from Lionel Wilson}

BECAUSE compounds are generally much more soluble in magmas at the high pressures of their mantle source regions than at the surface, volcanoes generally release gases. But the rates of gas release are difficult to quantify. Thanks, however, to the excellent long-term monitoring programme of the US Geological Survey's Hawaiian Volcano Observatory, T.M. Gerlach and E.J. Graeber of the Sandia National Laboratories have been able to estimate the volatile budget of Kilauea, most active of the Hawaiian volcanoes, over the past 27 years. Their results are published on page 273 of this issue.

Like many volcanoes, Kilauea has a high-level magma storage zone, consisting of a network of dykes and sills. Parental basaltic magma, rising at a fairly constant rate from deep mantle sources, is either erupted directly to the summit area or stored causing inflation of the volcanic edifice. If direct eruption takes place, as commonly occurred before 1924 , the released gases will be typical of the volatile content of the source regions at depth. Gerlach and Graeber, who call these volatiles Type I gases, suggest that a sample of such gases has only once been collected without significant contamination by air or atmospheric water (by J.A. Jaggar in 1917-18). This sample contained about $59 \%$ carbon dioxide, $18 \%$ water vapour and $10 \%$ sulphur (as sulphur dioxide) by weight, with traces of carbon monoxide, hydrogen, hydrogen sulphide and hydrogen chloride.

Since 1924, direct eruption of parental magma at Kilauea has been rare; instead, magma is stored in the summit reservoir, exsolving volatiles until equilibrium is reached with the local pressure. Presumably these excess volatiles either leak to the surface or become involved in chemical reactions, perhaps in near-surface hydrothermal systems. Intermittently, magma migrates sideways into the east or south-west rift zones to erupt at distances of up to tens of kilometres from the summit. The amounts of volatiles (termed Type II gases) released at the vent represent the difference between the equilibrium states in the storage zone and at the surface. Samples of these gases (collected by several scientists in recent years) consist of about $69 \%$ water vapour, $22 \%$ sulphur (mainly as sulphur dioxide) and $8 \%$ carbon dioxide by weight, with small amounts of hydrochloric acid, hydrogen fluoride, hydrogen and traces of atmospheric gases.

Knowing the relative amounts of these volatile compounds in Types I and II gases is, however, only the first stage of establishing the absolute amounts released by the erupting magma. The key link comes from a comparison of the sulphur contents of three types of erupted material: samples of spatter from vents on land; samples of lava erupted underwater from vents forming an off-shore extension of the east rift zone; and glass inclusions in olivine crystals forming part of the erupted magma.

The spatter samples represent droplets of magma which have had ample opportunity to release all their excess volatiles while forming part of a fire-fountain above the vent. The submarine lavas, on the other hand, have been subjected to water pressures high enough to cause them to retain essentially all of the volatiles present in the storage zone. Finally, the glasses trapped inside olivine crystals should have had no opportunity to release any volatiles since leaving the parental magma source in the mantle. Thus, the difference between the sulphur contents of the spatter samples and the submarine lavas should give the absolute weight fraction of the sulphur lost by the magma into Type II gases, and the difference between the sulphur contents of spatter samples and glass inclusions should give the absolute amount of sulphur lost to Type I gases. It is then a simple matter to convert the ratios of the amounts (relative to sulphur) of the other compounds that are present in the gases to their absolute weight fractions.

From the well-documented volumes of lava erupted from Kilauea in Type I and Type II activity since 1956, Gerlach and Graeber calculate a total volatile budget which shows that, on average, anthropo- genic inputs of carbon dioxide and sulphur dioxide to the atmosphere exceed those of the volcano by factors of 15,000 and 380 , respectively. It would, of course, be very valuable to have the same kind of information for other commonly-active volcanic centres, so that a global budget could be calculated, but several factors conspire to make this difficult. First, the physical collection of uncontaminated gas samples near volcanic vents is never easy; remote-sensing methods of gas analysis using spectroscopic methods are effective but have difficulty dealing with the minor components of the mixture. Second, whereas Kilauea fortuitously provides lava samples in submarine eruptions which are typical of the magma in high-level storage, in general such material is not available. (The alternative technique of analysing glass inclusion in magmatic crystals for evidence of the preeruption magma chemistry is becoming accepted as reliable, but there is sometimes considerable ambiguity as to the depth in the volcanic system at which the entrapment took place.) Third, the Hawaiian Volcano Observatory monitors the volumetric output of Kilauea with a thoroughness that is seldom matched elsewhere; and, finally, many volcanoes show a greater variation of magma chemistry with time than does Kilauea, so that it would require reliable data from every eruption for calculation of an accurate budget.

Gerlach and Graeber's work has underlined what can be done by careful study. Only with strong financial support for volcano monitoring will it be possible to carry out this kind of analysis of other active centres.

Lionel Wilson is in the Department of Envrionmental Science, University of Lancaster, Lancaster LAI 4YQ, U.K.

\section{Psychophysics Structured representation in low-level vision}

from R. J. Watt

BEFORE we can claim to understand vision, we must provide explanations of the ability to discriminate and the appearance of visual stimuli that go beyond being simply descriptions of the stimuli themselves. At the centre of our gaze (the fovea), vision is usually so faithful to the stimuli that its operations are well concealed. But on page 308 of this issue Rentscher and Treutwein show that this is not the case outside the fovea, where, it seems, much less powerful representations are constructed ${ }^{1}$.

Rentschler and Treutwein have compared the abilities of foveal and peripheral vision instantly to discriminate pairs of different spatially periodic stimuli. Specifically, the stimuli are constructed by superimposing a cosine wave grating with its third harmonic, as shown in the figure. For each pair of stimuli, the relative phases of the fundamental and third harmonic differ by $120^{\circ}$ around a mean phase difference of $0^{\circ}$, $90^{\circ}$ or $130^{\circ}$ (so the relative phases of the pairs are: $-60^{\circ},+60^{\circ} ;+30^{\circ},+150^{\circ}$; $+120^{\circ},+240^{\circ}$ ). Only the pair around $90^{\circ}$ differ in contrast profile; the other two pairs are mirror images of each other. Although each pair is discriminated equally well when seen in foveal vision, in peripheral vision the $90^{\circ}$ pair is discriminated much more easily than the mirrorsymmetrical pairs.

Rentschler and Treutwein interpret this result as implying that the relative position of local image components or features is lost in peripheral, but not in foveal vision. 